\title{
Asymptotic analysis of a bit brace shaped junction of thin rods
}

\section{Leugering, Günter}

2019-11-07

Leugering , G , Nazarov , S A , Slutskij , A S \& Taskinen , J 2019 , ' Asymptotic analysis of a bit brace shaped junction of thin rods ' , Zeitschrift für Angewandte Mathematik und Mechanik , vol. 100 , no. 1 , 201900227 . https://doi.org/10.1002/zamm.201900227

http://hdl.handle.net/10138/327104

https://doi.org/10.1002/zamm.201900227

acceptedVersion

Downloaded from Helda, University of Helsinki institutional repository.

This is an electronic reprint of the original article.

This reprint may differ from the original in pagination and typographic detail.

Please cite the original version. 


\title{
Asymptotic analysis of a bit brace shaped junction of thin rods
}

\author{
Günter Leugering, Sergei A. Nazarov, Andrey S. Slutskij, Jari Taskinen*
}

October 8, 2019

\begin{abstract}
We present a 1-D model of a junction of five thin elastic rods forming the shape of a bit brace (hand drill), or, a crankshaft. The distinguishing feature of this junction is the existence of the socalled movable elements, which are rods and knots requiring modifications of the classical asymptotic ansätze. These consist of constant longitudinal displacements on the edges of the skeleton of the junction and affect the transmission conditions at its nodes. We provide asymptotic formulas for the displacements, stresses and elastic energy, as well as error estimates. An exact solution of the model is given for a particular loading.
\end{abstract}

1. Motivation. The theory of thin elastic rods, including the cases of isotropic and anisotropic, homogeneous and heterogeneous, straight and slightly curved ones, is generally considered as being completed both from the point of view of theory and applications. It is remarkable that many different mathematical tools have been developed for this important special problem in elasticity theory. The theoretical research relevant to the present work consists of error estimates with respect to Sobolev or Hölder norms, which are based on anisotropic weighted Korn inequalities and constructions of boundary layers ${ }^{1}$.

Rod elements and their junctions can be found in almost all engineering and architectural objects as well as in our biological and abiotic environment. However, there are only few publications on the systems of rods in comparison with the huge literature dealing with isolated rods, see the monographs and papers $[2,3,4,5,6,7]$ and the references in them.

An L-shaped joint of two thin isotropic bars of relative thickness $h \ll 1$ was considered in the pioneering paper [8]. The bars were assumed to have equal rectangular cross-sections and to be perpendicular to each other; either clamped or free ends were allowed. The author derived a 1-D model of the circumscribed elastic junction and justified the model by proving the so-called convergence theorem related to the limit passage $h \rightarrow+0$ in the rescaled variational formulation of the elasticity problem, see [8] and the monograph [9].

Another approach, based on anisotropic Korn type inequalities of [12], was developed in [10] and [11], see also the review paper [13]. These papers contain asymptotic analysis realized as the dimension reduction procedure, which produces 1-D models for thin 2-D beams and 3-D rods joined into a connected structure of an arbitrary shape. The main idea of this analysis is the classification of fixed/movable rods and nodes. In this way one identifies what we call the movable parts of the rod junction.

The movable parts require a modification of the classical asymptotic ansätze on thin rods. It is necessary to introduce additional algebraic unknowns so that the longitudinal and transversal displacements of a movable rod $\mathcal{Q}^{k}(h)$ get the same order in the small parameter $h$ and become, respectively,

$$
h^{-2} a_{k}+h^{-1} w_{3}^{k}\left(z^{k}\right) \text { and } h^{-2} w_{i}^{k}\left(z^{k}\right), i=1,2,
$$

where the notation and normalization factors of Section 2 are used. For a fixed rod, the constant $a_{k}$ is always null. For a movable one, there is a clear reason to introduce the term $h^{-2} a_{k}$, which is

\footnotetext{
*Acknowledgements. The research of the second named author was partially supported by the Russian Science Foundation, project no. 17-11-01003, and the Academy of Finland, project no. 308829. The research of the third named author was partially supported by Russian Foundation of Basic Research, project no. 18-01-00325. The research of the fourth named author was partially supported by the grant from the Faculty of Science of University of Helsinki.

${ }^{1}$ As shown in [1], the investigation of the boundary layer phenomenon near the rod ends is necessary for the derivation of pointwise error estimates for strains and stresses.
} 
accompanied by the second term $h^{-1} w_{3}^{k}$ depending on the longitudinal coordinate $z^{k}$ and being of higher order (because $w_{3}^{k}$ is of mean zero, see (3.3) and (3.2), (3.4)). Furthermore, this modification leads to the new phenomenon of non-local transmission conditions, which are related to far-actions of longitudinal forces applied at the ends of a movable rod (cf. formula (4.13) and comments to it).

The identification of the movable parts and the concomitant derivation of the transmission conditions in the 1-D model are rather intricate, and their recursive, algorithmic description in $[10,11]$ is cumbersome and difficult to present in a completely general situation. However, in the paper [5] the authors examined in detail the simplest, $\mathrm{V}$-shaped junction of two beams with an arbitrary angle between the two rods, "legs". It was shown that the effect of the non-local transmission conditions depends on the boundary conditions imposed on the "soles" of the legs. In the present paper we consider the 3-D junction of five rods (Fig. 1) with fixed, right angles between them. One knot $\left(G_{1}(h)\right)$ and one rod $\left(Q_{1}(h)\right)$ are fixed, but

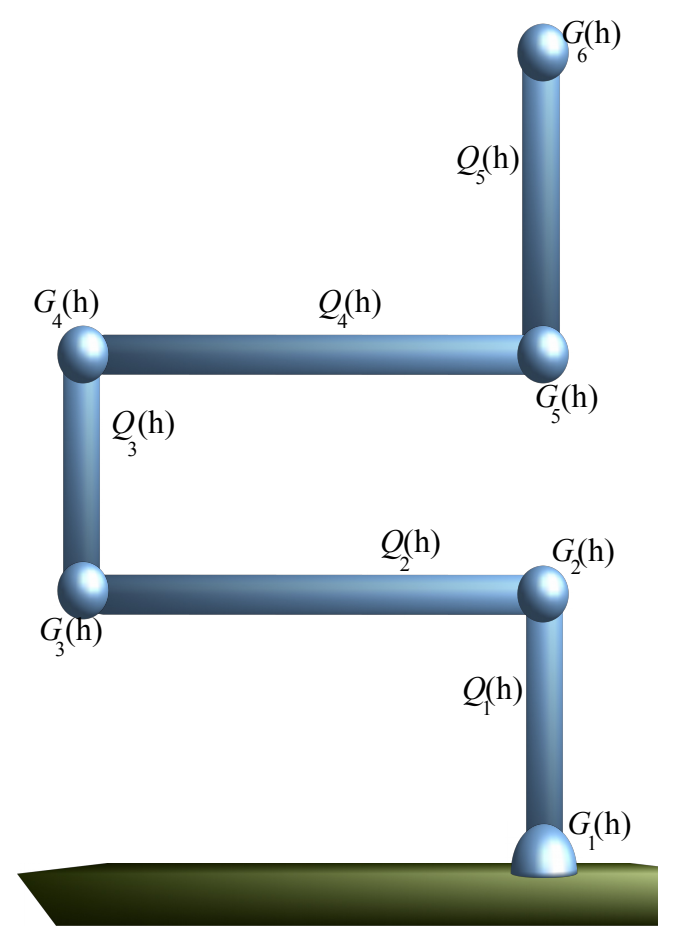

Figure 1: A bit brace $\Omega(h)$.

the other four rods and five knots of the junction may have longitudinal shifts of high order with respect to $h$. We will explain the classification procedure by means of a direct comparison of the asymptotic ansätze on neighbouring rods. As a result we obtain the stable transmission conditions, while the intrinsic transmission conditions will be derived by using the 1-D Green formula for the limit system of ordinary differential equations. This approach to thin elastic junctions looks very much simpler than that in [10], [11] and can thus be applied to more complicated configurations, like the crankshaft (see Fig. 2).

In Section 2 we will state the elasticity problem in detail. In order to simplify the proofs we assume that all rods are straight circular cylinders (so that the asymptotic formulas for the distribution of the stresses in the rods become explicit, well-known and simple). Notice that no restriction on the shape of the knots is needed because they play a secondary role in the derivation of the model; however, it is important to identify them as movable or fixed. We also present an example of a load which imitates the usual working condition of a bit brace.

In Section 3 we will outline the classical asymptotic formulas for stresses and strains in isolated elastic rods and the ordinary differential equations describing their deformations. The transmission conditions are derived in Section 4 by a method which is a reduction and reshaping of the procedure in [11]. In 


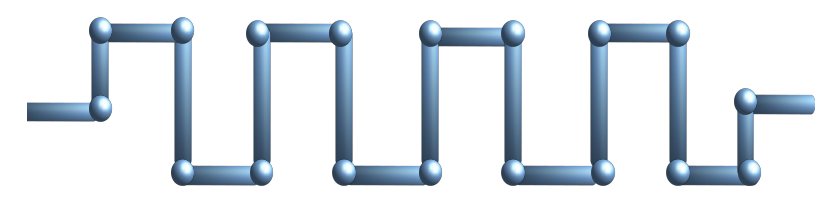

Figure 2: A crankshaft.

Section 5 we compute the exact solution of the 1-D model and in Section 6 we present error estimates which are based on the general results in [10], [11].

2. Formulation of the three-dimensional elasticity problem. We consider the junction $\Omega(h) \subset$ $\mathbb{R}^{3}$ of five thin rods $\mathcal{Q}^{k}(h)$, which are straight cylinders of length $2 l_{k}$, posed in right angles between them as in Fig. 1. We set the global Cartesian coordinate system $x=\left(x_{1}, x_{2}, x_{3}\right)$ of $\mathbb{R}^{3}$ such that the origin $O$ coincides with the midpoint $P_{1}$ (see below) of the first end of the first rod $\mathcal{Q}^{1}(h)$ and the $x_{3}$-axis is directed along the axis of the $\operatorname{rod} \mathcal{Q}^{1}(h)$. Moreover, we associate to each $\operatorname{rod} \mathcal{Q}^{k}(h), k=1, \ldots, 5$, the (local) Cartesian coordinates $x^{k}=\left(x_{1}^{k}, x_{2}^{k}, x_{3}^{k}\right)=\left(y_{1}^{k}, y_{2}^{k}, z^{k}\right)$, which are connected to the global system by

$$
x^{k}=\mathcal{O}^{k}(x)-P_{k}
$$

where the mappings $\mathcal{O}^{k}$ are rotations of $\mathbb{R}^{3}$ and $P_{2}=\left(0,0,2 l_{1}\right), P_{3}=\left(0,-2 l_{2}, 2 l_{1}\right), P_{4}=\left(0,-2 l_{1}, 2\left(l_{1}+\right.\right.$ $\left.\left.l_{3}\right)\right), P_{5}=\left(0,0,2\left(l_{1}+l_{3}\right)\right), P_{6}=\left(0,0,2\left(l_{1}+l_{2}+l_{3}\right)\right)$ in the global coordinates. For $j=1,2,3$, we denote by $e_{j}$ (respectively, $\left.e_{j}^{k}, k=1, \ldots, 5\right)$ the canonical basis vectors of the global (resp. $k$ th local) coordinate system. We assume the following relations between the directions in the local and global coordinates:

$$
e_{j}^{k}=e_{j}, k=1,3,5 ; \quad e_{1}^{2}=e_{1}, e_{2}^{2}=-e_{3}, e_{3}^{2}=e_{2} ; \quad e_{1}^{3}=e_{1}, e_{3}^{2}=e_{3}, e_{3}^{3}=-e_{2} .
$$

This choice of coordinate axes determines the rotations $\mathcal{O}^{k}$, which actually differ from the identity mapping only for $k=2,4$. In the local coordinates, the rods are directed along the $z^{k}$-axes so that they can be presented as

$$
\mathcal{Q}^{k}(h)=\left\{\left(y^{k}, z^{k}\right) \in \mathbb{R}^{3}: z^{k} \in \Upsilon^{k}=\left(-l_{k}, l_{k}\right), \eta^{k}=h^{-1} y^{k} \in \omega_{k}\right\}, \quad k=1, \ldots, 5,
$$


Here, $y^{k}=\left(y_{1}^{k}, y_{2}^{k}\right)$ are the coordinates on the planes perpendicular to the axes and $\omega_{k}=\left\{y^{k} \in \mathbb{R}^{2}\right.$ : $\left.\left|y^{k}\right|<r_{k}\right\}$ are discs of radius $r_{k}>0, k=1, \ldots, 5$, while the relative thickness $h$ belongs to the interval $\left(0, h_{0}\right]$, where $h_{0}>0$ is small. By rescaling we reduce the minimal length $\min \left\{l_{1}, \ldots, l_{5}\right\}$ to 1 .

The "skeleton" $\mathfrak{S}$ of the junction $\Omega(h)$ consists of the line segments $\overline{\Upsilon^{k}}$ connecting the points $P_{k}$ and $P_{k+1}, k=1, \ldots, 5$, see Fig. 3. These points are also the centers of the nodes $\mathcal{G}_{h}^{k}$, defined by

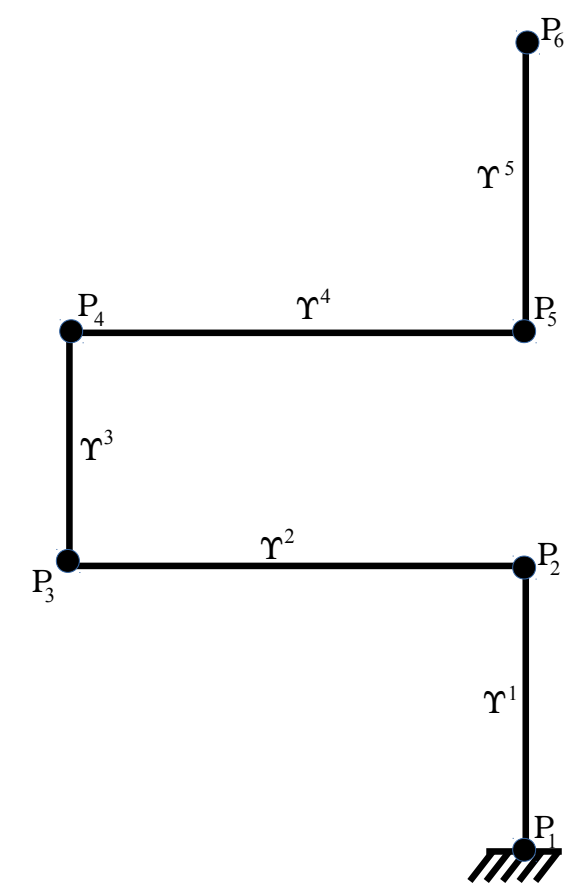

Figure 3: The "skeleton" $\mathfrak{S}$ of the bit brace $\Omega(h)$.

$$
\mathcal{G}_{h}^{k}=\left\{x \in \mathbb{R}^{3}: h^{-1}\left(x-P_{k}\right) \in \mathcal{G}_{1}^{k}\right\}, \quad k=1, \ldots, 6,
$$

where $\mathcal{G}_{1}^{k} \in \mathbb{R}^{3}$ are some fixed bounded domains with piecewise smooth boundary surfaces and independent of $h$.

The bit brace is defined as the set

$$
\Omega(h)=\bigcup_{k=1}^{5} \mathcal{Q}^{k}(h) \cup \bigcup_{m=1}^{6} \mathcal{G}_{h}^{m},
$$

and we assume that the downward force $h^{-1} e_{3} F(x)$ is applied to the subset

$$
\gamma_{h}=\partial \mathcal{G}_{h}^{6} \backslash \overline{\mathcal{Q}^{5}(h)}
$$

of the surface of the uppermost node $\mathcal{G}_{h}^{6}$ ("handle") and the twisting force $e_{1} f\left(\eta^{3}, z^{3}\right)$ affects the surface

$$
s_{h}=\left\{\left(y^{3}, z^{3}\right): z^{3} \in \Upsilon^{3}, \eta^{3}=h^{-1} y^{3} \in \partial \omega_{3}\right\} \backslash\left(\mathcal{G}_{3}^{h} \cup \mathcal{G}_{4}^{h}\right)
$$

of the central rod $\mathcal{Q}^{3}(h)$ ("crank"), respectively (see Fig. 1). Note that the factor $h^{-1}$ of the downward force makes these two forces affect by the same order of magnitude. Then, the deformation of the isotropic homogeneous solid $\Omega(h)$ is described by the boundary value problem

$$
-\mu \Delta u^{h}-(\lambda+\mu) \nabla \nabla \cdot u^{h}=0 \text { in } \Omega(h),
$$




$$
\begin{gathered}
\sigma^{(n)}\left(u^{h}\right)=e_{3} h^{-1} F \text { on } \gamma_{h} \subset \partial \mathcal{G}_{h}^{6}, \\
\sigma^{(n)}\left(u^{h}\right)=e_{1} f \text { on } s_{h}, \\
\sigma^{(n)}\left(u^{h}\right)=0 \text { on } \Omega(h) \backslash\left(\Gamma_{h} \cup s_{h} \cup \gamma_{h}\right), \\
u^{h}=0 \text { on } \Gamma_{h} \subset \partial \mathcal{G}_{h}^{1},
\end{gathered}
$$

where $\nabla$ denotes the gradient, $\nabla \cdot$ the divergence and $\Delta$ the Laplace operator, and

$$
\Gamma_{h}=\left\{x \in \partial \mathcal{G}_{h}^{1}: x_{3}=0\right\},
$$

which is assumed to be a set of positive 2-D Lebesgue measure. Moreover, $\lambda \geqslant 0$ and $\mu>0$ are the Lamé constants, $u^{h}=\left(u_{1}^{h}, u_{2}^{h}, u_{3}^{h}\right)$ is the displacement vector, and stresses are determined by

$$
\begin{gathered}
\sigma_{p q}\left(u^{h}\right)=\mu\left(\frac{\partial u_{q}^{h}}{\partial x_{p}}+\frac{\partial u_{p}^{h}}{\partial x_{q}}\right)+\delta_{p, q} \lambda\left(\frac{\partial u_{1}^{h}}{\partial x_{1}}+\frac{\partial u_{2}^{h}}{\partial x_{2}}+\frac{\partial u_{3}^{h}}{\partial x_{3}}\right), \\
\sigma^{(n)}\left(u^{h}\right)=\left(\sigma_{1}^{(n)}, \sigma_{2}^{(n)}, \sigma_{3}^{(n)}\right), \quad \sigma_{p}^{(n)}=n_{1} \sigma_{p 1}+n_{2} \sigma_{p 2}+n_{3} \sigma_{p 3},
\end{gathered}
$$

where, $\delta_{p, q}$ is the Kronecker symbol and $n=\left(n_{1}, n_{2}, n_{3}\right)$ is the outward normal unit vector. Finally, volume forces like gravity are not taken into account, since these are negligible in comparison with the much bigger surface forces applied to the bit brace.

3. Asymptotic ansätze. The following asymptotic forms for the displacement field $u$ on the rods $\mathcal{Q}^{k}(h)$ are suggested by the traditional dimension reduction procedure:

$$
u^{h}(x) \sim h^{-2} U_{(k)}^{-2}\left(z^{k}\right)+h^{-1} U_{(k)}^{-1}\left(\eta^{k}, z^{k}\right)+h^{0} U_{(k)}^{0}(\eta, z)+h^{1} U_{(k)}^{1}\left(\eta^{k}, z^{k}\right)+\ldots
$$

Here, $U_{(k)}^{p}$ are vector functions on $\mathcal{Q}^{k}(1)$ to be determined. The factors $h^{-q}$ of $U_{(k)}^{-q}$ are posed so that the stresses and strains gain the order $h^{-1}$ while the elastic energy stored in the junction (2.4) becomes $O(1)$ as $h \rightarrow+0$. However, even the main terms differ from the traditional form of the case of the isolated rod. In the terminology [11] the rods $\mathcal{Q}^{k}(h), k=2,3,4,5$ are now movable and, as it was mentioned in Section 1 , the main term $U_{(k)}^{-2}$ is assumed to be of the form

$$
U_{(k)}^{-2}\left(z^{k}\right)=w_{1}^{k}\left(z^{k}\right) e_{1}^{k}+w_{2}^{k}\left(z^{k}\right) e_{2}^{k}+a_{k} e_{3}^{k}, \quad k=2,3,4,5,
$$

where the coefficients $w_{1}^{k}$ and $w_{2}^{k}$ are functions and $a_{k}$ is a constant. Only on the clamped rod $\mathcal{Q}^{1}(h)$ we keep the conventional form of the main term

$$
U_{(1)}^{-2}\left(z^{1}\right)=w_{1}^{1}\left(z^{1}\right) e_{1}^{1}+w_{2}^{1}\left(z^{1}\right) e_{2}^{1} .
$$

Apparently, the difference of this and (3.2) consists of setting $a_{1}=0$ as a consequence of the Dirichlet condition (2.9). As was demonstrated in [10], [11] and mentioned in Section 1, the introduction of the constant $a_{k}$ into the main term (3.2) requires the orthogonality condition

$$
\int_{-l_{k}}^{l_{k}} w_{3}^{k}\left(z^{k}\right) d z^{k}=0, \quad k=2,3,4,5
$$

in the next term (3.2). For the clamped $\operatorname{rod} \mathcal{Q}^{1}(h)$ the restriction (3.4) is not posed. Since the constants $a_{k}$ in (3.2) are eliminated in the differentiation, the structure of the asymptotic terms $U_{(k)}^{q}$ with $q \geqslant-1$ is the same for all $k$ :

$$
U_{(k)}^{-1}\left(\eta^{k}, z^{k}\right)=\left(w_{3}^{k}\left(z^{k}\right)-\sum_{i=1}^{2} \eta_{j}^{k} \partial_{z} w_{j}^{k}\left(z^{k}\right)\right) e_{3}^{k}+w_{4}^{k}\left(z^{k}\right) \theta\left(\eta^{k}\right),
$$


Here, $\partial_{z}^{p}=\partial^{p} / \partial z^{p}$ and $z=z^{k}$ with any $k=1, \ldots, 5 ; w_{3}^{k}$ and $w_{4}^{k}$ are unknown functions and $\theta\left(\eta^{k}\right)=$ $2^{-1 / 2}\left(e_{1}^{k} \eta_{2}^{k}-e_{2}^{k} \eta_{1}^{k}\right)$ denotes the rotation around the axis of the rod.

The calculation of the next term in (3.1) is well-known and can be found in [3, 6, 7] and others. The term is obtained by finding the exact solutions in the unit disc for the 2-D elasticity system and for the Neumann Laplacian. We just reproduce these results and, consequently, define the third terms of the asymptotic ansätze as

$$
\begin{gathered}
\left(U_{(k)}^{0}\right)_{j}\left(\eta^{k}, z^{k}\right)=\frac{\lambda}{4(\lambda+\mu)}\left(\left(\left(\eta_{j}^{k}\right)^{2}-\left(\eta_{3-j}^{k}\right)^{2}\right) \partial_{z}^{2} w_{j}^{k}\left(z^{k}\right)+2 \eta_{1}^{k} \eta_{2}^{k} \partial_{z}^{2} w_{3-j}^{k}\left(z^{k}\right)\right. \\
\left.-2 \eta_{j}^{k} \partial_{z} w_{3}^{k}\left(z^{k}\right)\right), j=1,2, \quad\left(U_{(k)}^{0}\right)_{3}\left(\eta^{k}, z^{k}\right)=0
\end{gathered}
$$

To determine the terms $U_{(k)}^{1}$ and $U_{(k)}^{2}$ of the expansion (3.1), we pose for the functions $w_{p}^{k}$ the equations

$$
\begin{aligned}
& -\mathbf{m}_{j}^{k} \partial_{z}^{4} w_{j}^{k}\left(z^{k}\right)=\delta_{3, k} \delta_{1, j} \mathbf{f}^{k}\left(z^{k}\right), \quad j=1,2, \\
& -\mathbf{m}_{3}^{k} \partial_{z}^{2} w_{3}^{k}\left(z^{k}\right)=0,-\mathbf{m}_{4}^{k} \partial_{z}^{2} w_{3}^{k}\left(z^{k}\right)=0, \quad z^{k} \in\left(-l_{k}, l_{k}\right),
\end{aligned}
$$

where $\mathbf{f}^{k}=0$ for $k=1,2,4,5$,

$$
\mathbf{m}_{1}^{k}=\mathbf{m}_{2}^{k}=\frac{1}{4} \pi r_{k}^{4} E, \quad \mathbf{m}_{3}^{k}=\pi\left(r_{k}\right)^{2} E, \quad \mathbf{m}_{4}^{k}=\frac{1}{4} \pi r_{k}^{4} \mu, \quad \mathbf{f}^{3}\left(z^{3}\right)=\int_{s_{h}} e_{1} f\left(\eta^{3}, z^{3}\right) d s_{\eta^{3}}
$$

and $E=\frac{\mu(3 \lambda+2 \mu)}{\lambda+\mu}$ is Young's modulus. One can write system (3.7) in the form

$$
-\mathbf{D}\left(\partial_{z}\right)^{\top} \mathbf{M}^{k} \mathbf{D}\left(\partial_{z}\right) \vec{w}^{k}\left(z^{k}\right)=\mathbf{F}^{k}\left(z^{k}\right), \quad z^{k} \in\left(-l_{k}, l_{k}\right)
$$

where $\vec{w}^{k}=\left(w_{1}^{k}, w_{2}^{k}, w_{3}^{k}, w_{4}^{k}\right), \mathbf{D}\left(\partial_{z}\right)=\left(\partial_{z}^{2}, \partial_{z}^{2}, \partial_{z}, \partial_{z}\right)^{\top}$ and

$$
\mathbf{M}^{k}=\operatorname{diag}\left(\mathbf{m}_{1}^{k}, \mathbf{m}_{2}^{k}, \mathbf{m}_{3}^{k}, \mathbf{m}_{4}^{k}\right)^{\top}, \quad \mathbf{F}^{k}\left(z^{k}\right)=\left(\mathbf{f}^{k}\left(z^{3}\right), 0,0,0\right)^{\top} .
$$

4. Variational formulation of the problem. By a weak solution of the problem (2.5)-(2.9) in the Sobolev space $H^{1}\left(\Omega_{h}\right)^{3}$ (the superscript 3 indicates the number of the components) we understand an element of the subspace $H_{0}^{1}\left(\Omega_{h}\right)^{3}=\left\{u^{h} \in H^{1}\left(\Omega_{h}\right)^{3}: u^{h}=0\right.$ on $\left.\Gamma_{h}\right\}$ satisfying the integral identity

$$
2 \mathbf{E}\left(u^{h}, \varphi^{h} ; \Omega_{h}\right)-\left(e_{1} f, \varphi^{h}\right)_{s_{h}}-h^{-1}\left(e_{3} F, \varphi^{h}\right)_{\gamma_{h}}=0 \quad \forall \varphi^{h} \in H_{0}^{1}\left(\Omega_{h}\right)^{3},
$$

where $(\cdot, \cdot) \Xi$ is the natural inner product in the Lebesque space $L^{2}(\Xi)$ for the given domain $\Xi$ and

$$
\begin{gathered}
\mathbf{E}\left(u^{h}, \psi^{h} ; \Omega_{h}\right)=\frac{1}{2} \sum_{j, k=1}^{3}\left(\sigma_{j k}\left(u^{h}\right), \varepsilon_{j k}\left(\psi^{h}\right)\right)_{\Omega_{h}} \\
=\frac{\lambda}{2}\left(\sum_{j=1}^{3} \varepsilon_{j j}\left(u^{h}\right), \sum_{j=1}^{3} \varepsilon_{j j}\left(\psi^{h}\right)\right)_{\Omega_{h}}+\mu \sum_{j=1}^{3} \sum_{k=1}^{4-j}\left(\varepsilon_{j k}\left(u^{h}\right), \varepsilon_{j k}\left(\psi^{h}\right)\right)_{\Omega_{h}}, \\
\mathbf{E}\left(u^{h}, u^{h} ; \Omega_{h}\right)=\int_{\Omega_{h}}\left(\frac{\lambda}{2}\left(\sum_{j=1}^{3} \varepsilon_{j j}\left(u^{h}\right)\right)^{2}+\mu \sum_{j=1}^{3} \sum_{k=1}^{4-j} \varepsilon_{j k}\left(u^{h}\right)^{2}\right) d x .
\end{gathered}
$$

To derive an integral identity for the limit system (3.8), we replace in (4.1) the displacement field $u^{h}$ by the sum of first three terms in the ansätze (3.1) and take a test vector function $\varphi^{h}$, which is of the similar form

$$
\varphi^{h}(x)=h^{-2} \Phi_{(k)}^{-2}\left(z^{k}\right)+h^{-1} \Phi_{(k)}^{-1}\left(\eta^{k}, z^{k}\right)+h^{0} \Phi_{(k)}^{0}(\eta, z), \quad x \in \mathcal{Q}^{k}(h) .
$$


Here, the vector functions $\Phi_{(k)}^{q}$ are defined by formulas (3.2), (3.5), (3.6), where arbitrary vector functions $\psi^{k}=\left(\psi_{1}^{k}, \psi_{2}^{k}, \psi_{3}^{k}, \psi_{4}^{k}\right) \in C^{\infty}\left[-l_{k}, l_{k}\right]^{4}$ and constants $b^{k}$ replace the functions $w_{j}^{k}$ and the constants $a_{k}$. However, the fields $\Phi_{(k)}^{q}$ are properly defined on the rods (2.2) only, and in order to match and glue them on the nodes (2.3) it is necessary to impose boundary and transmission conditions at the skeleton nodes, according to the general procedure in [11].

First, we associate the Dirichlet conditions

$$
w_{j}^{1}\left(-l_{1}\right)=0, \partial_{z} w_{j}^{1}\left(-l_{1}\right)=0, w_{2+j}^{1}\left(-l_{1}\right)=0, \quad j=1,2,
$$

to the clamped knot $\mathcal{G}_{h}^{1}$ at the end of the rod $\mathcal{Q}^{1}(h)$. Next, we set the four triples of relations

$$
\begin{gathered}
\partial_{z} w_{1}^{k}\left(-l_{k}\right)+\alpha_{k-1} w_{4}^{k-1}\left(l_{k-1}\right)=0, \quad \partial_{z} w_{2}^{k}\left(-l_{k}\right)-\partial_{z} w_{2}^{k-1}\left(l_{k-1}\right)=0, \\
w_{4}^{k}\left(-l_{k}\right)-\alpha_{k-1} \partial_{z} w_{1}^{k-1}\left(l_{k-1}\right)=0,
\end{gathered}
$$

where $\alpha_{1}=\alpha_{4}=1$ and $\alpha_{2}=\alpha_{3}=-1$, which describe the continuity of rotation fields in the knots $\mathcal{G}_{h}^{m}, m=2,3,4,5$. Finally, in the same way, the continuity of the translations in these knots require the following relations:

$$
\begin{gathered}
w_{2}^{2}\left(-l_{2}\right)=0 ; \quad w_{1}^{p}\left(l_{p}\right)=w_{1}^{p+1}\left(-l_{p+1}\right), p=1,2,3,4 ; \\
w_{2}^{p}\left(l_{p}\right)=(-1)^{p+1} a^{p+1}, p=1,2,3,4 ; \quad w_{2}^{q}\left(-l_{q}\right)=(-1)^{q+1} a^{q-1}, q=3,4,5 .
\end{gathered}
$$

The equalities (4.5)-(4.7) are obtained by comparing the main terms (3.2), (3.3) of the different ansätze (3.1) with $k=m$ and $k=m+1$ on the $\operatorname{rod} \mathcal{Q}^{m}(h)$. We mention that in [11] the corresponding equations were derived by analysing the boundary layer phenomenon.

In the terminology of [14], the conditions (4.5)-(4.7) are called stable because they are inherited by the variational formulation of the limit problem, as explained after (4.1). Hence, the vector functions $\psi^{k}$ and the constants $b^{k}$ must be subject to (4.5)-(4.7) and the orthogonality condition (3.4) as well.

Computing the strains and stresses generated by the displacement field (4.3) yields

$$
\begin{gathered}
\varepsilon_{i i}=h^{-1} \frac{\lambda}{2(\lambda+\mu)}\left(\eta_{1} \partial_{z}^{2} \psi_{1}^{k}(z)+\eta_{2} \partial_{z}^{2} \psi_{2}^{k}(z)-\partial_{z} \psi_{3}^{k}(z)\right)+\ldots, \sigma_{i i}=\sigma_{12}=\sigma_{21}=0+\ldots \\
\varepsilon_{i 3}=\varepsilon_{3 i}=h^{-1}(-1)^{i+1} \frac{\sqrt{2}}{2} \eta_{i} \partial_{z} \psi_{4}^{k}(z)+\ldots, \sigma_{i 3}=\sigma_{3 i}=h^{-1}(-1)^{i+1} \sqrt{2} \mu \eta_{i} \partial_{z} \psi_{4}^{k}(z)+\ldots \\
\varepsilon_{12}=\varepsilon_{21}=0+\ldots, \quad \varepsilon_{33}=h^{-1}\left(\partial_{z} \psi_{3}^{k}(z)-\eta_{1} \partial_{z}^{2} \psi_{1}^{k}(z)-\eta_{2} \partial_{z}^{2} \psi_{2}^{k}(z)\right) \ldots \\
\sigma_{33}=E h^{-1}\left(\partial_{z} \psi_{3}^{k}(z)-\eta_{1} \partial_{z}^{2} \psi_{1}^{k}(z)-\eta_{2} \partial_{z}^{2} \psi_{2}^{k}(z)\right) \ldots
\end{gathered}
$$

where $i=1,2$ and dots stand for terms of order $h^{m}$ with $m \geqslant 0$. We insert the expressions (4.8) into the formula (4.1) and pass to the limit $h \rightarrow+0$ : according to [11], the contribution of the knots $\mathcal{G}_{h}^{m}$ is negligible, because the vector functions $\Phi_{(k)}^{q}$ have now been glued appropriately using (4.5)-(4.7) As a result we obtain the 1-D integral identity

$$
\begin{gathered}
\sum_{k=1}^{5}\left(\sum_{j=3}^{4} \mathbf{m}_{j}^{k} \int_{-l_{k}}^{l_{k}} \partial_{z} w_{j}^{k}\left(z^{k}\right) \partial_{z} \psi_{j}^{k}\left(z^{k}\right) d z^{k}-\sum_{j=1}^{2} \mathbf{m}_{j}^{k} \int_{-l_{k}}^{l_{k}} \partial_{z}^{2} w_{j}^{k}\left(z^{k}\right) \partial_{z}^{2} \psi_{j}^{k}\left(z^{k}\right) d z^{k}\right) \\
+\int_{-l_{k}}^{l_{k}} \mathbf{f}\left(z^{3}\right) \psi_{1}^{3} d z^{k}+F b^{5} \operatorname{mes}_{2} \gamma_{1}=0
\end{gathered}
$$

which is our 1-D model for the elastic junction (2.4); here, mes $_{2}$ denotes the 2-D Lebesgue measure.

We search for a solution of the variational problem $(4.9)$ in the space $\mathcal{H}(\mathfrak{S})$ of the vectors

$$
\begin{gathered}
\overrightarrow{\boldsymbol{\psi}}=\left(\psi^{1} ; \psi^{2}, b^{2} ; \ldots, ; \psi^{5}, b^{5}\right) \\
\in H^{2}\left(-l_{1}, l_{1}\right)^{2} \times H^{1}\left(-l_{1}, l_{1}\right)^{2} \times \prod_{k=2}^{5}\left(H^{2}\left(-l_{k}, l_{k}\right)^{2} \times H^{1}\left(-l_{k}, l_{k}\right)^{2} \times \mathbb{R}^{1}\right)
\end{gathered}
$$


satisfying the Dirichlet conditions (4.4), the transmission conditions (4.5), (4.6), (4.7) and the orthogonality conditions (3.4). A weak solution of the limit problem is an element $\vec{w}$ of the space $\mathcal{H}(\mathfrak{S})$ for which the identity (4.9) holds for all test vector functions $\overrightarrow{\boldsymbol{\psi}} \in \mathcal{H}(\mathfrak{S})$. The solvability of the limit problem has been proved in [10, Lemma 3.2], according to which the problem (4.9) indeed has a unique solution $\vec{w} \in \mathcal{H}(\mathfrak{S})$.

The weak formulation (4.9) of the 1-D model involves the so-called intrinsic transmission conditions as well, see [14]. To derive them we start by the Green formula

$$
\begin{gathered}
\sum_{k=1}^{6}\left(\int_{-l_{k}}^{l_{k}}\left(\mathbf{M}^{k} \mathbf{D}\left(\partial_{z}\right) \vec{w}^{k}\left(z^{k}\right) \mathbf{D}\left(\partial_{z}\right) \psi^{k}\left(z^{k}\right)+\psi_{1}^{3}\left(z^{3}\right) \mathbf{f}\left(z^{3}\right)\right) d z^{k}+\sum_{j=1}^{2}\left(\mathbf{m}_{j}^{k} \partial_{z}^{3} w_{j}^{k}\left(l_{k}\right) \psi_{j}^{k}\left(l_{k}\right)\right.\right. \\
\left.-\left(1-\delta_{k, 6}\right) \mathbf{m}_{j}^{k} \partial_{z}^{3} w_{j}^{k}\left(-l_{k}\right) \psi_{j}^{k}\left(-l_{k}\right)-\mathbf{m}_{j}^{k} \partial_{z}^{2} w_{j}^{k}\left(l_{k}\right) \partial_{z} \psi_{j}^{k}\left(l_{k}\right)+\left(1-\delta_{k, 6}\right) \mathbf{m}_{j}^{k} \partial_{z}^{2} w_{j}^{k}\left(-l_{k}\right) \partial_{z} \psi_{j}^{k}\left(-l_{k}\right)\right) \\
-\sum_{j=3}^{4}\left(\mathbf{m}_{j}^{k} \partial_{z} w_{j}^{k}\left(l_{k}\right) \psi_{j}^{k}\left(l_{k}\right)-\left(1-\delta_{k, 6}\right) \mathbf{m}_{j}^{k} \partial_{z} w_{j}^{k}\left(-l_{k}\right) \psi_{j}^{k}\left(-l_{k}\right)\right)+b^{5} F \operatorname{mes}_{2} \gamma=0 .
\end{gathered}
$$

Because of the ODE-system (3.8), the integral term is cancelled in (4.11). We next consider the other sums on each knot $\mathcal{G}_{h}^{q}, q=1, \ldots, 6$, of the junction and use the stable conditions in order to find relations which annul these terms for any test vector (4.10).

First of all, the terms with $k=1$ vanish because $\psi_{j}^{1}$ meets the Dirichlet conditions (4.4). Then, let us consider the node $\mathcal{G}_{h}^{6}$. The term

$$
\begin{gathered}
\mathbf{m}_{1}^{5} \psi_{1}^{5}\left(l_{5}\right) \partial_{z}^{3} w_{1}^{5}\left(l_{5}\right)+\mathbf{m}_{2}^{5} \psi_{2}^{5}\left(l_{5}\right) \partial_{z}^{3} w_{2}^{5}\left(l_{5}\right)-\mathbf{m}_{4}^{5} \psi_{4}^{5}\left(l_{5}\right) \partial_{z} w_{4}^{5}\left(l_{5}\right) \\
-\mathbf{m}_{1}^{5} \partial_{z} \psi_{1}^{5}\left(l_{5}\right) \partial_{z}^{2} w_{1}^{5}\left(l_{5}\right)-\mathbf{m}_{2}^{5} \partial_{z} \psi_{2}^{5}\left(l_{5}\right) \partial_{z}^{2} w_{2}^{5}\left(l_{5}\right)
\end{gathered}
$$

is annulled, if and only if

$$
\mathbf{m}_{1}^{5} \partial_{z}^{3} w_{1}^{5}\left(l_{5}\right)=\mathbf{m}_{2}^{5} \partial_{z}^{3} w_{2}^{5}\left(l_{5}\right)=0, \mathbf{m}_{4}^{5} \partial_{z} w_{4}^{5}\left(l_{5}\right)=0, \mathbf{m}_{1}^{5} \partial_{z}^{2} w_{1}^{5}\left(l_{5}\right)=\mathbf{m}_{2}^{5} \partial_{z}^{2} w_{2}^{5}\left(l_{5}\right)=0 .
$$

These equalities imply he cancellation of the transversal forces $\mathbf{m}_{j}^{5} \partial_{z}^{3} w_{2}^{5}\left(l_{5}\right), j=1,2$, the bending moments $\mathbf{m}_{j}^{5} \partial_{z}^{2} w_{2}^{5}\left(l_{5}\right), j=1,2$, and the torque moment $\mathbf{m}_{4}^{5} \partial_{z} w_{4}^{5}\left(l_{5}\right)$. According to (4.7) for $\overrightarrow{\boldsymbol{\psi}}$ (see (4.10)) we have $b^{5}=\psi_{2}^{4}\left(l_{4}\right)$ and, therefore, removing the corresponding terms in (4.11) yields

$$
\mathbf{m}_{2}^{4} \partial_{z}^{3} w_{2}^{4}\left(l_{4}\right)+F \operatorname{mes}_{2} \gamma=0 .
$$

We observe that the introduction of the algebraic unknowns in the model transfers the longitudinal force acting at the node $\mathcal{G}_{h}^{6}$ along the $\operatorname{rod} \mathcal{Q}^{5}(h)$ and converts it into a transversal force applied to $\mathcal{Q}^{4}(h)$. A reason for such a transformation is that the order $h^{-1}$ of the surface force loading in (2.6) is too large to be compensated by the term $w_{3}^{5}$ in (3.5). Similar effects occur for the other couples of the knots, see below.

Consider the knot $\mathcal{G}_{h}^{5}$. The equilibrium of the forces acting in directions $e_{j}, j=1,3$ implies the equalities

$$
\mathbf{m}_{1}^{5} \partial_{z}^{3} w_{1}^{5}\left(-l_{5}\right)-\mathbf{m}_{1}^{4} \partial_{z}^{3} w_{1}^{4}\left(l_{4}\right)=0, \text { for } j=1 ; \quad \mathbf{m}_{3}^{5} \partial_{z} w_{3}^{5}\left(-l_{5}\right)=0 \text { for } j=3 .
$$

Furthermore, the equilibrium of the moments leads to

$$
\begin{gathered}
\mathbf{m}_{1}^{5} \partial_{z}^{2} w_{1}^{5}\left(-l_{5}\right)-\mathbf{m}_{2}^{4} \partial_{z}^{2} w_{2}^{4}\left(l_{4}\right)=0, \quad \mathbf{m}_{2}^{5} \partial_{z}^{2} w_{2}^{5}\left(-l_{5}\right)-\mathbf{m}_{4}^{4} \partial_{z} w_{4}^{4}\left(l_{4}\right)=0, \\
\mathbf{m}_{4}^{5} \partial_{z} w_{4}^{5}\left(-l_{5}\right)+\mathbf{m}_{1}^{4} \partial_{z}^{2} w_{1}^{4}\left(l_{4}\right)=0 .
\end{gathered}
$$

The equalities $\psi_{2}^{3}\left(l_{3}\right)=b^{4}, b^{4}=\psi_{2}^{5}\left(-l_{5}\right)$, contained in (4.7)) for $\overrightarrow{\boldsymbol{\psi}}$ ], lead to the nonlocal relation $\psi_{2}^{3}\left(l_{3}\right)=\psi_{2}^{5}\left(-l_{5}\right)$, which gives the following nonlocal transmission condition for longitudinal forces:

$$
\mathbf{m}_{2}^{5} \partial_{z}^{3} w_{2}^{5}\left(-l_{5}\right)=\mathbf{m}_{2}^{3} \partial_{z}^{3} w_{2}^{3}\left(l_{3}\right)
$$


Analogously to the above, the equilibrium of the forces and moments acting at $\mathcal{G}_{h}^{k}, k=2,3,4$, yield the following transmission conditions:

$$
\begin{gathered}
\mathbf{m}_{1}^{k+1} \partial_{z}^{3} w_{1}^{k+1}\left(-l_{k+1}\right)-\mathbf{m}_{1}^{k} \partial_{z}^{3} w_{1}^{k}\left(l_{k}\right)=0, k=2,3 ; \quad \mathbf{m}_{3}^{k} \partial_{z} w_{3}^{k}\left( \pm l_{k}\right)=0, k=2,3,4 \\
\mathbf{m}_{2}^{k+1} \partial_{z} w_{2}^{k+1}\left(-l_{k+1}\right)=\alpha_{k} \mathbf{m}_{2}^{k-1} \partial_{z}^{3} w_{2}^{k-1}\left(l_{k-1}\right), \quad k=2,3, \mathbf{m}_{2}^{2} \partial_{z} w_{2}^{2}\left(-l_{2}\right)=0
\end{gathered}
$$

and

$$
\begin{gathered}
\mathbf{m}_{1}^{k+1} \partial_{z}^{2} w_{1}^{k+1}\left(-l_{k+1}\right)-\alpha_{k} \mathbf{m}_{4}^{k} \partial_{z} w_{4}^{k}\left(l_{k}\right)=0, \quad \mathbf{m}_{2}^{k+1} \partial_{z}^{2} w_{2}^{k+1}\left(-l_{k+1}\right)-\mathbf{m}_{2}^{k} \partial_{z}^{2} w_{2}^{k}\left(l_{2}\right)=0, \\
\mathbf{m}_{4}^{k+1} \partial_{z} w_{4}^{k+1}\left(-l_{k+1}\right)+\alpha_{k} \mathbf{m}_{1}^{k} \partial_{z}^{2} w_{1}^{k}\left(l_{k}\right)=0, \quad k=2,3,4
\end{gathered}
$$

The formulas (4.12) - (4.18) form the intrinsic transmission conditions for the system (3.8).

It has been proved in [11] that the 1-D model is uniquely solvable, because of the Dirichlet conditions (4.4). The variational formulation of the model consists of the integral identity (4.9) posed in the space $\mathcal{H}(\mathfrak{S})$, while the differential formulation involves the system (3.8) of ordinary differential equations at the edges of the skeleton $\mathfrak{S}$, the boundary conditions (4.4) and the stable and intrinsic transmission conditions (4.4)-(4.7) and (4.12)-(4.18), respectively, at the nodes of $\mathfrak{S}$.

5. An exact solution to the 1D model. We finally consider the special case where the data $f$ does not depend on $z$ and derive the corresponding simplified formulas. We search for the components of the solution $\vec{w}$ of the problem (4.9) as the polynomial

$$
w_{j}^{k}\left(z^{k}\right)=-\delta_{j, 1} \delta_{k, 3}\left(z^{k}\right)^{4}\left(\mathbf{m}_{1}^{3}\right)^{-1} \mathbf{f} / 12+\sum_{p=0}^{3} C_{j p}^{k}\left(z^{k}\right)^{p}, w_{3}^{k}=0, w_{4}^{k}=C_{41}^{k} z^{k}+C_{40}^{k},
$$

We substitute (5.1) into the differential statement of the model and obtain a system with 54 linear equations for the 54 constants $C_{p q}^{k}$ and $a_{k}$. However, the number of the equations and unknowns is reduced to 50 by excluding the coefficients $a_{2}, a_{3}, a_{4}, a_{5}$, converting the relations (4.7) into

$$
\begin{gathered}
w_{2}^{1}\left(l_{1}\right)=w_{2}^{3}\left(-l_{1}\right), w_{2}^{2}\left(l_{2}\right)=w_{2}^{4}\left(-l_{1}\right), w_{2}^{3}\left(l_{2}\right)=w_{2}^{5}\left(-l_{1}\right), \\
a^{2}=w_{2}^{3}\left(-l_{1}\right), a^{3}=-w_{2}^{4}\left(-l_{1}\right), a^{4}=w_{2}^{3}\left(l_{2}\right), a^{5}=-w_{2}^{4}\left(l_{2}\right),
\end{gathered}
$$

from which the constants $a_{k}$ can be reconstructed.

To simplify the final formulas, we assume that rods are made of the same material and have the same radii, therefore $\mathbf{m}_{p}^{k}=\mathbf{m}_{p}, k=1,2,3,4,5$ and $r_{k}=r, k=1,2,3,4,5$. Let $l_{1}=l_{5}=H, l_{2}=l_{4}=l, l_{3}=L$.

We get

$$
\begin{gathered}
w_{1}^{1}(z)=8 \mathbf{f} L\left(z^{3}+3(H+L) z^{2}+3 H(2 H+3 L) z+\mathbf{c}_{1}\right), w_{2}^{1}(z)=0, w_{4}^{1}(z)=96 \mathbf{f} L l(z+H) \mathbf{p} \\
w_{1}^{2}(z)=-8 \mathbf{f} L\left(z^{3}+3 l z^{2}+3 l(3 l-8 H \mathbf{p}) z+\mathbf{c}_{2}\right), \\
w_{2}^{2}(z)=\mathbf{q} \mathbf{F}\left(z^{3}+3 l z^{2}+l^{2} z+l^{3}\right) / 6, w_{4}^{2}(z)=-48 \mathbf{f} L\left(L \mathbf{p} z+l L \mathbf{p}-2 H L-2 H^{2}\right) \\
w_{1}^{3}(z)=-2 \mathbf{f} L\left(2 z^{3}-3 L z^{2}+2\left(24 l L \mathbf{p}-7 L^{2}-24 H L-24 H^{2}\right) z+\mathbf{c}_{3},\right. \\
w_{2}^{3}(z)=l \mathbf{q} \mathbf{F}\left(z^{2}+2(L+l) z+L(L+2 l)\right), w_{4}^{3}(z)=96 l \mathbf{q} \mathbf{F}(2 H \mathbf{p}-l) ; \\
w_{1}^{4}(z)=96 l L \mathbf{f}(2 H \mathbf{p}-\mathbf{l}) z+\mathbf{c}_{4}, w_{2}^{4}(z)=-\mathbf{q} \mathbf{F}\left(z^{3}-3 l z^{2}-3 l(8 L+7 l) z-3 l^{2}(8 L+3 l) / 6\right. \\
w_{4}^{4}(z)=32 \mathbf{f}\left(3 l L^{2}-L^{3}-3 H L^{2}-3 H^{2} L\right) ; w_{1}^{5}(z)=32 \mathbf{f}\left(L^{3}+3 H L^{2}+3 H^{2} L-3 l L^{2}\right) z+\mathbf{c}_{5}, \\
w_{2}^{5}(z)=4 l \mathbf{q} \mathbf{F}(l+L), w_{4}^{5}(z)=96 \mathbf{f} l L(2 H-l),
\end{gathered}
$$


where

$$
\mathbf{F}:=\frac{1}{\mathbf{m}_{3}^{5}} \int_{\gamma_{1}} e_{3} F d s, \quad \mathbf{p}=\frac{\mathbf{m}_{1}}{\mathbf{m}_{4}}=\frac{3 \lambda+2 \mu}{\lambda+\mu}, \quad \mathbf{q}=\frac{\mathbf{m}_{2}}{\mathbf{m}_{3}}=\frac{r^{2}}{4} .
$$

The constants $\mathbf{c}_{k}$ in (5.4) are defined by

$$
\begin{gathered}
\mathbf{c}_{1}=H^{2}(5 H+3 L), \mathbf{c}_{2}=5 l^{3}+16 H^{3}+12 H^{2} L-14 l^{2} H \mathbf{p}, \\
\mathbf{c}_{3}=L \mathbf{f}\left(17 L^{3}+24\left(4 H L^{2}-16 H l^{2} \mathbf{p}-4 l L^{2} \mathbf{p}+8 H^{2} L\right)+128\left(H^{3}+l^{3}\right)\right), \\
\mathbf{c}_{4}=L \mathbf{f}\left(48 L^{3}+32\left(6 H L^{2}-6 H l^{2} \mathbf{p}-6 l L^{2} \mathbf{p}+9 H^{2} L+6 l^{3}+4 H^{3}\right)\right), \\
\mathbf{c}_{5}=8 L \mathbf{f}\left(6 L^{3}+28 H L^{2}-12 H l L \mathbf{p}-24 l L^{2} \mathbf{p}+48 H^{2} L+28 H^{3}-8 l^{3}\right) .
\end{gathered}
$$

According to (5.3) the constants $a_{k}$ in the ansätze (3.2) on the movable rods are

$$
a_{2}=0, \quad a_{3}=-\mathbf{q} \mathbf{F} l^{3}, \quad a_{4}=-a_{5}=4 \mathbf{q} \mathbf{F} l(l+L) .
$$

6. Concluding remarks. Formula (5.5) shows that even a modest loading of the bit brace (2.4) causes large longitudinal shifts of the rods $\mathcal{Q}^{3}(h), \mathcal{Q}^{4}(h)$ and $\mathcal{Q}^{5}(h)$. Although they do not influence on the stresses directly, they affect the whole solutions $\vec{w}$ through the transmission conditions which lead to the non-local stable conditions (5.2).

The introduction of the movable elements of the junctions also changes the error estimates for the 1-D models. Without going into the proofs, which are cumbersome and require the construction of the boundary layers (cf. [1]), we mention that

$$
\begin{gathered}
\left\|u_{i}^{h}-h^{-2}\left(U_{(1)}^{-2}\right)_{i} ; L^{2}\left(\mathcal{Q}^{1}(h)\right)\right\| \leqslant c h^{-1 / 2}, i=1,2, \\
\left\|u_{3}^{h}-h^{-1}\left(U_{(1)}^{-2}\right)_{3} ; L^{2}\left(\mathcal{Q}^{1}(h)\right)\right\| \leqslant c h^{1 / 2},
\end{gathered}
$$

where $U_{(1)}^{-2}$ and $U_{(1)}^{-1}$ are taken from formulas (3.3) and (3.5) for the clamped $\operatorname{rod} \mathcal{Q}^{1}(h)$, whereas for the movable rods $\mathcal{Q}^{k}(h), k=2, \ldots, 5$, there holds the modified estimates

$$
\begin{gathered}
\left\|u_{j}^{h}-h^{-2}\left(U_{(k)}^{-2}\right)_{j} ; L^{2}\left(\mathcal{Q}^{k}(h)\right)\right\| \leqslant c h^{-1 / 2}, j=1,2,3, \\
\left\|\left(u_{3}^{h}\right)_{\perp}-h^{-1}\left(U_{(k)}^{-1}\right)_{3} ; L^{2}\left(\mathcal{Q}^{k}(h)\right)\right\| \leqslant c h^{1 / 2},
\end{gathered}
$$

where $U_{(k)}^{-2}, U_{(k)}^{-1}$ are taken from $(3.2),(3.5)$ and $\perp$ denotes the projection onto the subspace of functions of zero mean in the Lebesgue space $L^{2}\left(\mathcal{Q}^{k}(h)\right)$. Note that $\left(U_{(k)}^{-1}\right)_{3}$ belongs to this subspace, due to our assumption on the symmetry and the orthogonality condition (3.4).

The error estimates for the stresses and strains and, therefore, for the elastic energy (4.2) look the same as for isolated rods, namely

$$
\left\|\sigma_{p q}\left(u^{h}\right)-h^{-1} S_{p q}^{k}\left(w^{k}\right) ; L^{2}\left(\mathcal{Q}^{k}(h)\right)\right\| \leqslant c h^{1 / 2},
$$

where $S_{p q}^{k}\left(w^{k}\right)$ are the coefficients of $h^{-1}$ in formulas (4.8) for the stresses with the change $\psi^{k} \mapsto w^{k}$. Furthermore,

$\left|\mathbf{E}\left(u^{h}, u^{h} ; \Omega(h)\right)-\pi \sum_{k=1}^{5} \int_{-l / 2}^{l / 2}\left(A\left(r_{k}^{4}\left(\left(\partial_{z}^{2} w_{1}^{k}\right)^{2}+\left(\partial_{z}^{2} w_{2}^{k}\right)^{2}\right)+4 r_{k}^{2}\left(\partial_{z} w_{3}^{k}\right)^{2}\right)+\mu \frac{r_{k}^{4}}{4}\left(\partial_{z} w_{4}^{k}\right)^{2}\right) d z\right| \leqslant c h^{1 / 2}$,

where

$$
A=\frac{(\lambda+\mu)\left(3 \lambda^{2}+4 \lambda \mu+2 \mu^{2}\right)}{16(\lambda+\mu)^{2}} .
$$

Finally, all of the above presented estimates involve integral norms. According to [1], in order to derive pointwise estimates for elastic fields in junctions of thin elastic structures it is necessary to take into account the boundary layer phenomenon, which is not considered in the present paper. 


\section{References}

[1] S.A. Nazarov, Justification of the asymptotic theory of thin rods. Integral and pointwise estimates, Probl. Mat. Anal. St.-Petersburg: St.-Petersburg Univ. 17 (1997) P. 101-152. (English transl.: J. Math. Sci. 97 (1999), N 4. P. 4245-4279)

[2] P.G. Ciarlet, Plates and Junctions in Elastic Multi-Structures: An Asymptotic Analysis. Masson, Paris, 1990.

[3] J. Sanchez-Hubert, É. Sanchez-Palencia, Introduction aux méthodes asymptotiques et à homogénéisation, Masson: Paris. 1992.

[4] J.E. Lagnese, G. Leugering, E.J.P.G. Schmidt, Modelling of Dynamic Networks of Thin Thermoelastic Beams, Math. Methods Appl. Sci. 16 (1993), 327-358

[5] G. Leugering, S.A. Nazarov, A.S. Slutskij, The asymptotic analysis of a junction of two elastic beams, Z. Angew. Math. Mech 2019 (to appear)

[6] S.A. Nazarov, Asymptotic Theory of Thin Plates and Rods. Vol. 1. Dimension Reduction and Integral Estimates [in Russian], Novosibirsk: Scientific Books Publisher (IDMI), 2002.

[7] G.P. Panasenko, Multi-Scale Modelling for Structures and Composites. Springer 2005.

[8] H. Le Dret, Modeling of the junction between two rods, J. Math. Pures Appl. 68 (1989). P.365-397.

[9] H. Le Dret, Problèmes variationnels dans les multi-domains. Modélisation des jonctions et applications, Rech. Math. Appl., 19 (Masson, Paris, 1991).

[10] S.A. Nazarov, A.S. Slutskii, Arbitrary plane systems of anisotropic beams, Tr. Mat. Inst. Steklova, 236, 234-261 (2002) (English transl.: Proc. Steklov Inst. Math., 236(1), 222-249 (2002).))

[11] S.A. Nazarov, A.S. Slutskii, Asymptotic analysis of an arbitrary spatial system of thin rods, Trudy St.-Petersburg Mat. Obshch. 10, 63-115 (2004), (English transl.: Trans. Am. Math. Soc. Ser. 2. 214, 59-108 (2005)).

[12] Nazarov S.A., Slutskii A.S. Korn's inequality for an arbitrary system of thin curved rods, Sibirsk. Mat. Zh. 2002. V. 43, N 6. P. 1319-1331. (English transl.: Siberian Math. J. 2002. V. 43, N. 6. P. 1069-1079.)

[13] Nazarov S.A. Korn's inequalities for elastic junctions of massive bodies and thin plates and rods, Uspehi mat. nauk. 2008. V. 63, N 1. P. 37-110 (English transl.: Russ. Math. Surveys. 2008. V. 63, N 1. P. 35-107).

[14] J.-L. Lions, E. Magenes, Non-homogeneous boundary value problems and applications. Springer, Berlin, 1972.

Authors' addresses:

Department Mathematik, Lehrstuhl für Angewandte Mathematik 2, Cauerstr. 11, 91058 Erlangen, Germany

Saint-Petersburg State University, Universitetskaya nab. 7-9, St. Petersburg, 199034, Russia

Saint-Petersburg State University, Universitetskaya nab. 7-9, St. Petersburg, 199034, Russia

Department of Mathematics and Statistics, University of Helsinki, P.O.Box 68, 00014 Helsinki, Finland 